\title{
STREAMLINED BIOREACTOR-BASED PRODUCTION OF HUMAN CARTILAGE TISSUES
}

\author{
B. Tonnarelli ${ }^{\S}$, R. Santoro ${ }^{\S}$, M. Adelaide Asnaghi and D. Wendt* \\ Departments of Surgery and of Biomedicine, University Hospital Basel, Basel, Switzerland
}

$\S$ Equally contributing authors

\begin{abstract}
Engineered tissue grafts have been manufactured using methods based predominantly on traditional labourintensive manual benchtop techniques. These methods impart significant regulatory and economic challenges, hindering the successful translation of engineered tissue products to the clinic. Alternatively, bioreactor-based production systems have the potential to overcome such limitations. In this work, we present an innovative manufacturing approach to engineer cartilage tissue within a single bioreactor system, starting from freshly isolated human primary chondrocytes, through the generation of cartilaginous tissue grafts. The limited number of primary chondrocytes that can be isolated from a small clinicallysized cartilage biopsy could be seeded and extensively expanded directly within a 3D scaffold in our perfusion bioreactor ( $5.4 \pm 0.9$ doublings in 2 weeks), bypassing conventional 2D expansion in flasks. Chondrocytes expanded in 3D scaffolds better maintained a chondrogenic phenotype than chondrocytes expanded on plastic flasks (collagen type II mRNA, 18-fold; Sox-9, 11-fold). After this "3D expansion" phase, bioreactor culture conditions were changed to subsequently support chondrogenic differentiation for two weeks. Engineered tissues based on 3D-expanded chondrocytes were more cartilaginous than tissues generated from chondrocytes previously expanded in flasks. We then demonstrated that this streamlined bioreactor-based process could be adapted to effectively generate up-scaled cartilage grafts in a size with clinical relevance $(50 \mathrm{~mm}$ diameter). Streamlined and robust tissue engineering processes, as the one described here, may be key for the future manufacturing of grafts for clinical applications, as they facilitate the establishment of compact and closed bioreactor-based production systems, with minimal automation requirements, lower operating costs, and increased compliance to regulatory guidelines.
\end{abstract}

Keywords: Bioreactor, tissue engineering, proliferation, differentiation, scaffolds, chondrocyte, cartilage, culture $(2 \mathrm{D} / 3 \mathrm{D})$.

\section{*Address for correspondence:}

David Wendt

Institute for Surgical Research and Hospital Management University Hospital Basel

Hebelstrasse 20

4031 Basel, Switzerland

Telephone Number: 0041612653253

FAX Number: 0041612653990

E-mail: david.wendt@usb.ch

\section{Introduction}

Autologous chondrocyte implantation (ACI) and the second generation matrix-assisted ACI have long been established in the clinic for the repair of cartilage injuries. However, as these products are manufactured with minimal in vitro pre-cultivation times, they contain little to no extracellular matrix (ECM) and, therefore, lack the complex biological and mechanical signals which can be delivered by a more developed tissue graft.

Our laboratory has recently conducted a clinical trial based on engineered cartilage tissues containing abundant extracellular matrix, for treatment of cartilage lesions (Pelttari et al., 2014), with the ultimate goal of reducing rehabilitation time and generating a more durable long-term repair (Pelttari et al., 2009). While this clinical trial has demonstrated the safety, feasibility, and preliminary evidence of the efficacy of the tissue grafts, the manufacturing processes used to produce the engineered grafts were based on traditional bench-top manual culture methods. These manual procedures require a large number of labour-intensive manipulations that possess risks for contamination and introduce intra-/inter-operator variability, ultimately posing challenges towards regulatory compliance (Mason and Hoare, 2006). Moreover, the manual production of tissue grafts imparts limits with regards to up-scaling production volumes and ultimately results in high manufacturing costs in the long-term. As an alternative, bioreactor-based production systems, which automate and control the various bioprocesses, have the potential to overcome the limitations associated with conventional manufacturing methods (Martin et al., 2004; Ratcliffe and Niklason, 2002; Ratcliffe et al., 2011). However, a manufacturing process based on multiple independent and dedicated bioreactor systems (e.g. one to automate cell expansion and one to automate tissue culture) may be unable to demonstrate cost-benefit due to high development, capital, and operating costs. Ideally the entire manufacturing process would therefore be controlled within a single closed system, minimising any user interventions. However, the development of a fully automated and closed manufacturing system will likely require new and innovative concepts that not only replicate the often inefficient manual processes (e.g. by a robotic system), but instead simplify and streamline the conventional tissue engineering procedures (Martin et al., 2009).

In this work, we have developed an innovative manufacturing approach to produce engineered human cartilage tissue within a single bioreactor system, from the introduction of primary chondrocytes freshly isolated from a biopsy, until the generation of a cartilaginous tissue 

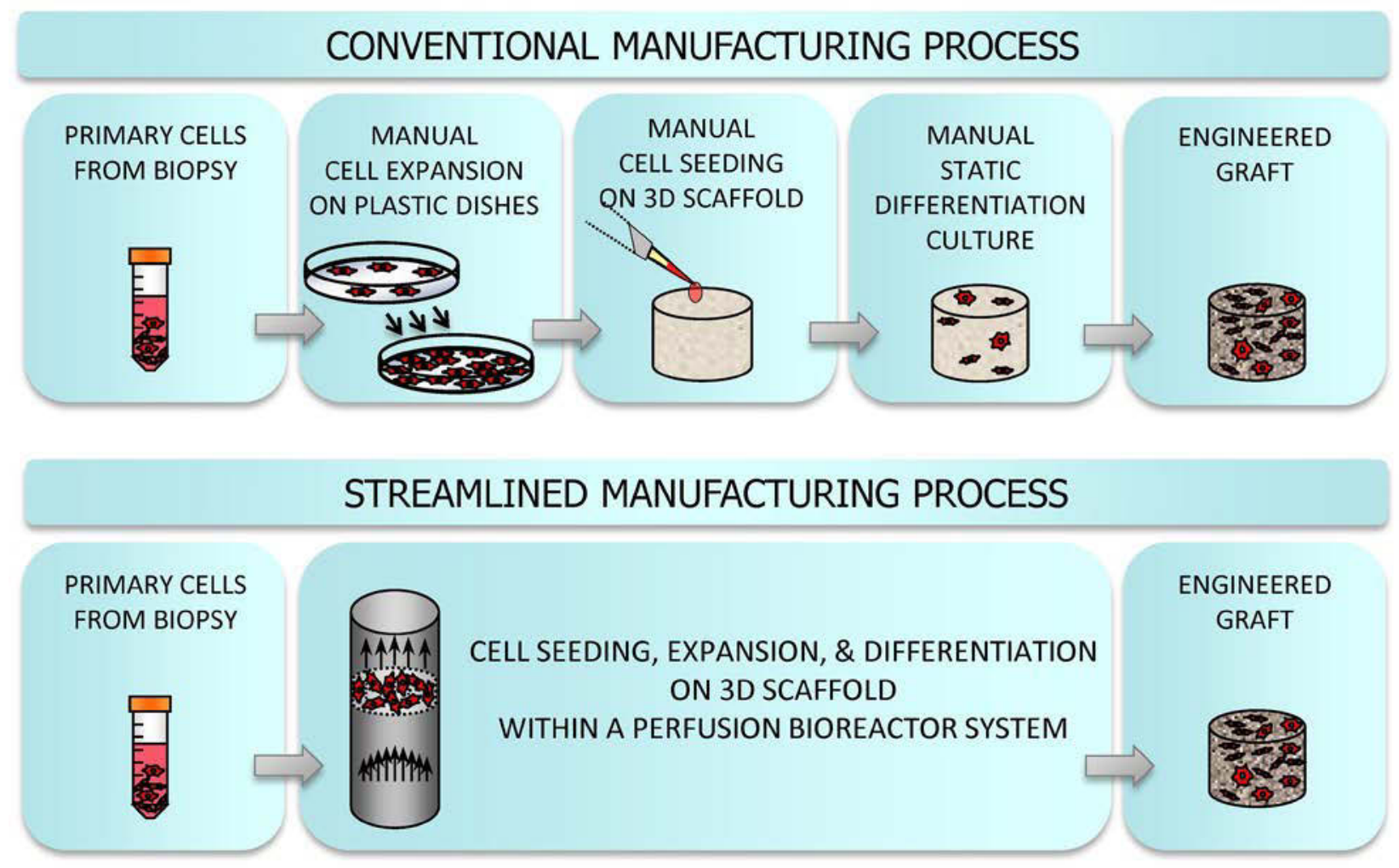

Fig. 1. The conventional approach to producing cell-based grafts has been based on traditional manual benchtop techniques. These procedures require a large number of manual and labour-intensive manipulations, ultimately posing significant challenges in terms of quality, safety, standardisation, regulatory compliance, and costs. As an alternative, we present a novel streamlined tissue engineering strategy to engineer cartilage tissue within a single bioreactor system, starting from freshly isolated primary chondrocytes, through the generation of a cartilaginous tissue graft. The simplified streamlined process facilitates the development of a simple, compact, and closed bioreactor design with minimal automation requirements, and with the likely result of lower operating costs and increased compliance to safety guidelines.

graft (Fig. 1). To validate this approach, we first aimed to determine whether a limited number of primary human chondrocytes, which can be isolated from a clinically relevant sized cartilage biopsy, could be extensively expanded directly within a 3D scaffold in a perfusion bioreactor, thereby eliminating the conventional labourintensive process of cell expansion in plastic dishes. Following this phase of "3D cell expansion", we next aimed to differentiate the 3D expanded cells within the bioreactor, to generate a cartilaginous tissue. Finally, we assessed whether this novel bioreactor-based cartilage engineering process could be effectively translated to the production of large-sized human cartilage grafts with clinically relevant dimensions $(50 \mathrm{~mm}$ diameter $\times 3 \mathrm{~mm}$ thick).

The streamlined process we describe here could serve as the basis of a cartilage graft manufacturing system that minimises the number of manual processes, thereby facilitating a simple, compact, and closed bioreactor design with limited automation requirements, and with the likely result of lower operating costs and increased compliance to safety guidelines.

\section{Materials and Methods}

\section{Isolation of primary chondrocytes}

Human articular cartilage samples were collected post mortem (within $24 \mathrm{~h}$ ), with informed consent and in accordance with the local Ethical Commission, from the knee joints of 8 donors (mean age $57 \pm 6$ years). Osteochondral samples (2-3 cm diameter, 1-2 cm thick) were dissected from the condyles of cadaveric joints using a surgical chisel. Cartilage tissues that macroscopically appeared healthy (i.e. white and smooth) were successively sliced from the subchondral bone with a scalpel, extensively rinsed with saline solution, and chopped into small pieces. Human articular chondrocytes (HAC) were isolated using $0.15 \%$ type II collagenase for $22 \mathrm{~h}$ and resuspended in Dulbecco's modified Eagle's medium ("high glucose" $25 \mathrm{mM}$ ) containing $10 \%$ foetal bovine serum, $4.5 \mathrm{mg} /$ $\mathrm{mL}$ D-glucose, $0.1 \mathrm{mM}$ nonessential amino acids, $1 \mathrm{mM}$ sodium pyruvate, $100 \mathrm{nM}$ Hepes buffer, $100 \mathrm{U} / \mathrm{mL}$ penicillin, $100 \mathrm{ug} / \mathrm{mL}$ streptomycin, and $0.29 \mathrm{mg} / \mathrm{mL}$ L-glutamine ("Complete Medium"). Primary HAC were counted (average $2.9 \pm 0.1 \times 10^{6}$ cells/gram of biopsy) and directly used for the following experimental groups. 
Table 1. Descriptions of the cell expansion phase and differentiation phase of the experimental groups.

\begin{tabular}{|l|c|c|c|}
\hline & 2D + Static & 2D + Perfusion & 3D + Perfusion \\
\hline Expansion Phase & $\begin{array}{c}\text { 2D expansion of cells } \\
\text { on plastic flasks }\end{array}$ & $\begin{array}{c}\text { 2D expansion of cells } \\
\text { on plastic flasks }\end{array}$ & $\begin{array}{c}\text { 3D expansion of cells } \\
\text { in scaffold under perfusion }\end{array}$ \\
\hline Differentiation Phase & $\frac{\text { Static culture of }}{\text { 3D construct }}$ & $\begin{array}{c}\text { Perfusion culture of } \\
\text { 3D construct }\end{array}$ & $\begin{array}{c}\text { Perfusion culture of } \\
\text { 3D construct }\end{array}$ \\
\hline
\end{tabular}

\section{Streamlined cartilage engineering process}

Primary HAC were seeded, expanded, and re-differentiated directly within a 3D scaffold using a perfusion bioreactor ("3D+Perfusion"; see Table 1) as follows. The bioreactor system was designed to first perfuse a cell suspension directly through the pores of a 3D scaffold, to seed cells uniformly throughout the entire scaffold (Wendt et al., 2003), and subsequently perfuse culture media, to maintain cell viability during expansion and re-differentiation (Wendt et al., 2006).

\section{Perfusion seeding phase}

A clinically relevant cell seeding density $\left(2 \times 10^{5}\right.$ cells/ $\mathrm{cm}^{3}$ scaffold volume) was calculated from the size of a cartilage biopsy obtained during ACI procedures (300$500 \mathrm{mg}$ ) (Brittberg et al., 1994), the average number of chondrocytes that can be obtained from a cartilage digest (2500 cells/mg cartilage) (Jakob et al., 2003), and an engineered graft of dimensions $50 \mathrm{~mm}$ diameter $\times 3 \mathrm{~mm}$ thick. A graft of this size would be sufficiently large to treat most focal cartilage defects of the femoral condyle, as well as for resurfacing joint facets such as the patella, trochlea, and tibial plateau of small to medium sized knee joints. In 5 independent experiments, $10^{4}$ freshly isolated primary $\mathrm{HAC}$ were injected into each bioreactor and perfusion seeded into Hyaff-1 $1{ }^{\circledR}$ non-woven meshes $(6 \mathrm{~mm}$ diameter $\times 2 \mathrm{~mm}$ thick; Anika Therapeutics, Abano Terme, Italy) at a perfusion rate of $1 \mathrm{~mm} / \mathrm{s}$ for $16 \mathrm{~h}$ (8 to 12 scaffolds per experiment). Hyaff-11 is a derivative of hyaluronic acid, a natural component of cartilage extracellular matrix. Hyalograft-C (autologous chondrocytes cultured on a Hyaff-11 mesh) was the first $3 \mathrm{D}$ cartilage tissue graft to be used clinically for the treatment of articular cartilage defects, and provided good mid-term clinical outcomes in young patients with single cartilage defects (Nehrer et al., 2009).

\section{D expansion phase}

Following perfusion seeding, cell-seeded constructs remained in the bioreactor system and were subsequently perfused with "proliferating" culture medium (Complete Medium, supplemented with $1 \mathrm{ng} / \mathrm{mL}$ transforming growth factor- $\beta 1$ (TGFß1), and $5 \mathrm{ng} / \mathrm{mL}$ fibroblast growth factor- 2 (FGF-2) (Jakob et al., 2001)), to expand the primary cells directly within the Hyaff-11 meshes. Constructs were perfused with $25 \mathrm{~mL}$ proliferating medium at a superficial velocity of $10 \mu \mathrm{m} / \mathrm{s}$ for 2 weeks with medium changes twice per week. Constructs were then either harvested and assessed biochemically, histologically, and for mRNA expression or remained in the bioreactor for further culture (re-differentiation phase).

\section{Re-differentiation phase}

Following the $3 \mathrm{D}$ expansion phase, constructs remained within the bioreactor and operating parameters were changed to support chondrocyte differentiation. The oxygen level of the incubator was reduced from $19 \%$ to $5 \% \mathrm{O}_{2}$, and constructs were perfused with "differentiating" culture medium (Complete Medium supplemented with $10 \mathrm{ng} / \mathrm{mL}$ TGFß1, $1 \mathrm{UI} / \mathrm{mL}$ insulin, and $0.1 \mathrm{mM}$ ascorbic acid 2-phosphate) (Moretti et al., 2005) for an additional 2 weeks. Constructs were then harvested and assessed biochemically, histologically, and for mRNA expression.

\section{Control cultures}

\section{$2 D$ expansion controls}

As controls, for each donor, freshly isolated primary HAC were also seeded onto conventional polystyrene tissue culture flasks and expanded in monolayer with proliferating medium for two passages. HAC were seeded at densities of $10^{4} \mathrm{HAC} / \mathrm{cm}^{2}$ for passage 1 and $5 \times 10^{3} \mathrm{HAC} /$ $\mathrm{cm}^{2}$ for passage 2. To assess the effects of the Hyaff-11 biomaterial on HAC expansion, freshly isolated primary HAC were seeded onto 2D membranes of Hyaff-11 (Anika Therapeutics) and expanded in monolayer with proliferating medium ( $n=2$ donors). The 2D membranes were thin, smooth, semi-permeable films composed of Hyaff-11.

\section{$3 D$ culture controls}

Following 2D expansion in flasks, HAC were seeded into $6 \mathrm{~mm}$ diameter $\times 2 \mathrm{~mm}$ thick Hyaff- 11 meshes either statically (Wendt et al., 2003) or under perfusion (Wendt et al., 2006), as previously described. For static seeding, chondrocytes were resuspended in $25 \mu \mathrm{L}$ of medium and slowly dispersed over the top surface of the scaffold with a micropipette. The seeded scaffolds were transferred to a $37^{\circ} \mathrm{C}$ incubator for $45 \mathrm{~min}$ to allow for initial cell attachment before carefully filling the wells of the 12well plate. The number of $2 \mathrm{D}$ expanded cells that was seeded into each mesh was equal to the average number of cells obtained following 2 weeks of $3 \mathrm{D}$ expansion in Hyaff-11 meshes (i.e., $5 \times 10^{5} \mathrm{HAC}$ ). In particular, in two experiments, scaffolds statically seeded with $2 \mathrm{D}$ expanded cells were subsequently cultured under conventional static conditions in Petri dishes ( $n=4$ per experiment) for 2 weeks in differentiating medium and under $5 \%$ oxygen ("2D+Static"; see Table 1). In two experiments, scaffolds perfusion-seeded with $2 \mathrm{D}$ expanded cells were subsequently cultured under perfusion in the bioreactor ( $n=4$ per experiment) for 2 weeks in differentiating medium and under $5 \%$ oxygen (" $2 \mathrm{D}+$ Perfusion"). 


\section{Streamlined engineered large-scale cartilage}

As proof-of-principle, we next assessed whether the streamlined cartilage engineering process could be effectively translated to the production of large-size human cartilage grafts with dimensions of clinical relevance. For this purpose, we used a perfusion bioreactor system previously developed for the engineering of large-scale human cartilage grafts, which was previously validated by seeding and re-differentiating a high density of $2 \mathrm{D}$ expanded HAC (Santoro et al., 2010). Using the upscaled bioreactor, $10^{6}$ freshly isolated primary HAC were perfusion seeded into a Hyaff-11 mesh $(50 \mathrm{~mm}$ diameter $\times 3 \mathrm{~mm}$ thick) at a superficial velocity of $1 \mathrm{~mm} / \mathrm{s}$ for $16 \mathrm{~h}$. Following perfusion seeding, constructs were perfused with proliferating culture medium ( $n=4$ experiments, $n=6$ constructs total) at a superficial velocity of $10 \mu \mathrm{m} / \mathrm{s}$. The number of cells within each $3 \mathrm{D}$ construct was nondestructively and non-invasively assessed throughout the proliferation phase using a method previously developed for small research-scale cartilage constructs, which was based on measurements of dissolved oxygen in the perfused culture medium (Santoro et al., 2011). Briefly, disposable chemo-optic flow-through micro-oxygen sensors (FTCPSt-3; PreSens GmbH, Regensburg, Germany) were connected to the top and bottom of the bioreactor scaffold chambers. Dissolved oxygen concentrations at the inlet and at the outlet of perfused constructs were measured every $10 \mathrm{~min}$. Based on the previously established linear correlation, the drop in oxygen across the perfused construct (i.e. $\Delta \mathrm{O}_{2}=\mathrm{C}_{\text {(Oxygen, inlet) }}-\mathrm{C}_{\text {(Oxygen, outlet) }}$ ) was then used to estimate the number of cells within each $3 \mathrm{D}$ construct. Using this method, instead of implementing a fixed endpoint based on culture time, the 3D expansion phase was continued in the bioreactor until the oxygen measurements predicted a total of $5 \times 10^{7}$ cells within the construct. This number of cells was based on the number of 2D expanded HAC seeded in the manufacture of cartilage grafts for clinical use (Albrecht et al., 2011). After reaching the target number of cells, constructs remained within the bioreactor and the oxygen tension and culture medium were changed to support chondrocyte differentiation ("Up-scaled 3D+Perfusion"; $n=2$ experiments, $n=2$ constructs total). As controls, in two experiments, largescale constructs were also generated using the conventional manual processes ("Up-scaled 2D+Static"). HAC were expanded for two passages in 2D monolayer culture and statically seeded into the large size Hyaff-11 meshes ( $5 \times 10^{7}$ expanded HAC per mesh). Cell seeded meshes were then cultured statically in dishes in differentiating medium and $5 \%$ oxygen for 2 weeks. Constructs were assessed histologically and biochemically. To assess the homogeneity of extracellular matrix deposition within the large-scale constructs, $6 \mathrm{~mm}$-diameter specimens were randomly punched from six locations and assessed biochemically.

\section{Construct characterisation}

\section{Histological and immunohistochemical analyses}

Constructs were fixed in $4 \%$ paraformaldehyde, dehydrated, and embedded in paraffin. $5 \mu \mathrm{m}$ sections were cut and analysed histologically and immunohistochemically.
Sections were stained with Safranin-O (SigmaAldrich, Buchs, Switzerland), with haematoxylin counterstaining, to visualise glycosaminoglycan (GAG). For immunohistochemical analysis, upon rehydration in an ethanol series, sections were subjected to enzymatic digestion (hyaluronidase and pronase). Sections were labelled with primary antibodies against collagen type I and type II (MP Biomedicals, Illkirch Cedex, France), and incubated for $2 \mathrm{~h}$ at room temperature. The immunobinding was detected with biotinylated secondary antibodies and by Vectastain ABC kit (Vector Laboratories, Peterborough, UK). The signal was developed with Vector Red Substrate kit (Vector Laboratories) as per manufacturer instructions, with haematoxylin as counterstaining. Negative controls were included for each analysis, omitting the primary antibodies.

\section{Biochemical analyses}

Engineered constructs were digested with protease $\mathrm{K}$ solution $(1 \mathrm{mg} / \mathrm{mL}$ protease $\mathrm{K}$ in $50 \mathrm{mM}$ Tris with $1 \mathrm{mM}$ EDTA, $1 \mathrm{mM}$ iodoacetamide, and $10 \mu \mathrm{g} / \mathrm{mL}$ pepstatin-A for $15 \mathrm{~h}$ at $56^{\circ} \mathrm{C}$ ) as previously described (Hollander et al., 1994). DNA was quantified with the CyQUANT ${ }^{\circledR}$ Cell Proliferation Assay Kit (Molecular Probes, Eugene, OR, USA), with calf thymus DNA as a standard. The number of population doublings during $3 \mathrm{D}$ expansion was calculated from the amount of DNA quantified in the engineered constructs after expansion and the amount of DNA quantified in an aliquot of the cell suspension that was initially seeded into the scaffold. GAG accumulated within the constructs was quantified with the dimethylmethylene blue colorimetric assay, with chondroitin sulphate as a standard (Farndale et al., 1986).

\section{Quantitative real-time RT-PCR}

Total RNA was extracted from cells using Trizol (Invitrogen, Carlsbad, CA, USA), treated with DNAse and retrotranscribed into cDNA, as previously described (Frank et al., 2002). Real-time reverse transcriptase-polymerase chain reaction (RT-PCR; 7300, Applied Biosystems, Waltham MA, USA) was performed as previously described (Barbero et al., 2003) to quantify expression levels of mRNA using the $\Delta \mathrm{Ct}$ method. $18 \mathrm{~S}$ was used as housekeeping gene to normalise the expression of the genes of interest, namely collagen type I ("CI"), collagen type II ("CII"), and SOX-9.

\section{Statistical analysis}

Values are represented as mean \pm standard deviation. Differences between the experimental groups were evaluated by non-parametric Mann Whitney U tests and considered to be statistically significant with $p \leq 0.05$.

\section{Results}

\section{HAC expansion}

Starting from the very low number of HAC that can be obtained from a clinically relevant sized cartilage biopsy, freshly isolated primary HAC could be extensively expanded directly within the 3D scaffold. During $7 \mathrm{~d}$ 

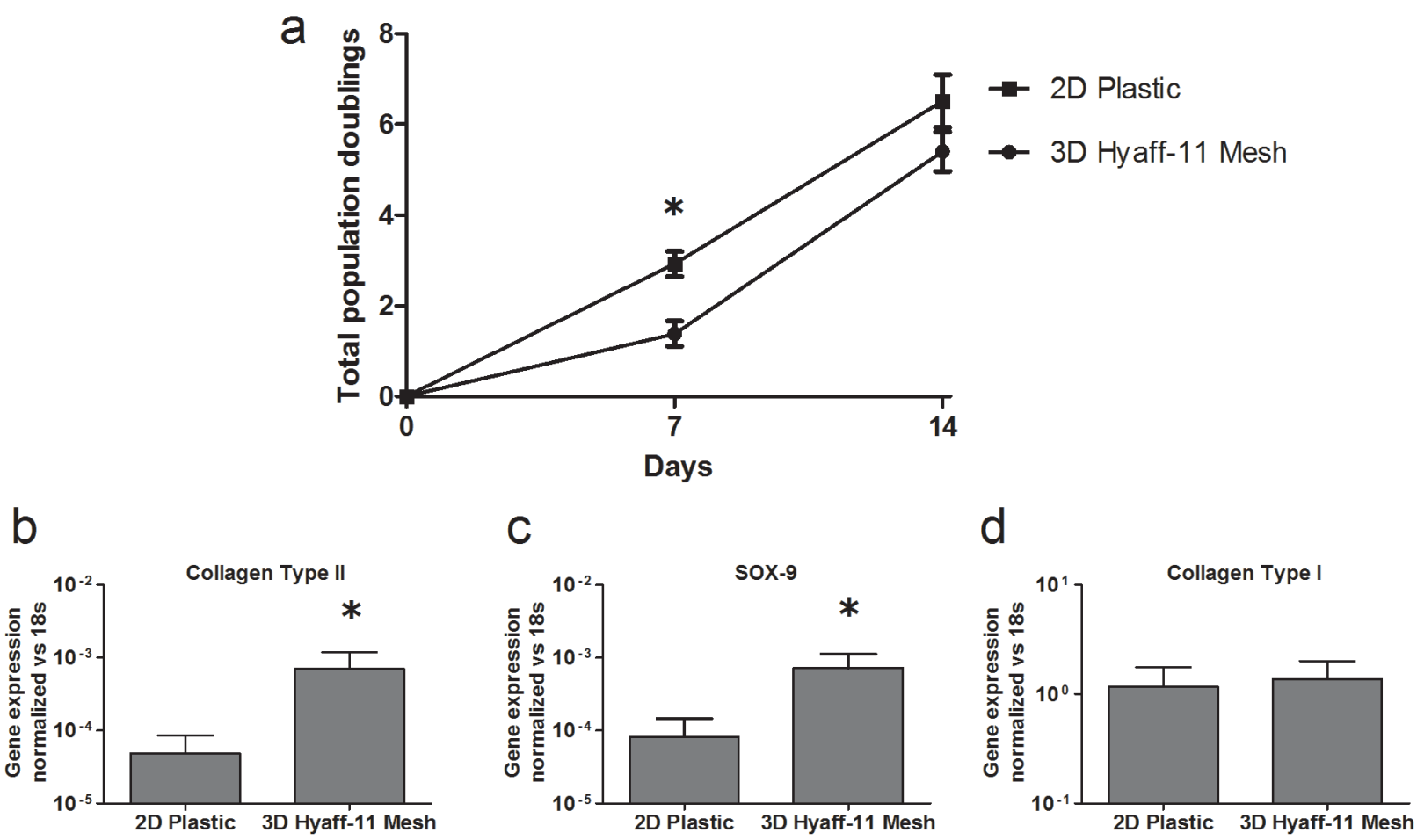

Fig. 2. Expansion of HAC in 3D Hyaff-11 meshes and on 2D plastic flasks. (a) Population doublings of HAC expanded directly within 3D Hyaff-11 meshes and on 2D plastic. (b,c,d) HAC expanded in 3D meshes had significantly higher mRNA expression levels of collagen type II and Sox-9, but similar expression levels of collagen type I as compared to HAC expanded in conventional $2 \mathrm{D}$ control cultures. * indicates statistical significance with $p \leq 0.05$.

a

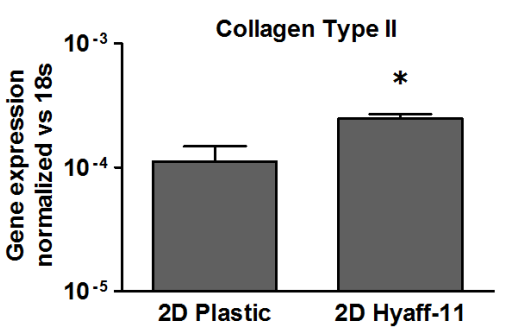

b

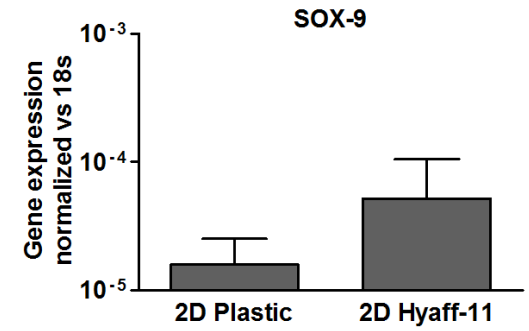

C

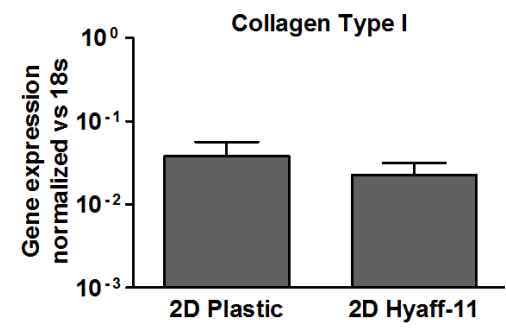

Fig. 3. HAC proliferation in 2D on plastic dishes and membranes of Hyaff-11. (a,b,c) HAC expanded on 2D Hyaff-11 membranes had significantly higher expression of collagen type II, slightly higher expression of Sox-9, but similar expression levels of collagen type I as compared to HAC expanded on conventional 2D plastic flasks. ${ }^{*}$ indicates statistical significance with $p \leq 0.05$.

of proliferation in the bioreactor, HAC expanded on 3D Hyaff-11 meshes underwent $1.4 \pm 0.6$ population doublings, which was significantly slower $(p=0.0075)$ than HAC expanded on 2D Plastic ( $2.9 \pm 0.6$ doublings) (Fig. 2a). However, after two weeks, the number of population doublings were not significantly different $(p=0.18)$ for HAC expanded on 3D Hyaff-11 meshes (5.4 \pm 0.9 doublings) or 2D Plastic ( $6.5 \pm 1.2$ doublings). HAC expanded on 3D Hyaff-11 meshes reached an average density of $7.4 \times 10^{6}$ cells $/ \mathrm{cm}^{3}$. HAC expanded in 3D Hyaff-11 meshes had significantly higher mRNA expression of collagen type II (18-fold, $p=0.050)$ and Sox-9 (11-fold, $p=0.050)$, but similar expression of collagen type I $(p=0.83)$, as compared to HAC expanded on conventional 2D plastic (Fig. 2b,c,d).
Expansion of primary HAC on 2D membranes of the Hyaff-11 biomaterial for 2 weeks yielded a heterogeneous population of cells with both rounded and fibroblasticlike morphologies. In contrast, HAC expanded on 2D plastic flasks were homogeneously fibroblastic-like in morphology. HAC expanded on membranes of 2D Hyaff-11 had 4-fold higher expression of collagen type II $(p=0.0495)$, but statistically insignificant differences in the expression of Sox-9 $(p=0.275)$ and collagen type I $(p=0.51)$ as compared to HAC expanded on conventional 2D plastic (Fig. 3).

\section{HAC differentiation}

Following 2 weeks of 3D expansion and 2 weeks of differentiation in the bioreactor, the $3 \mathrm{D}+$ Perfusion 
a

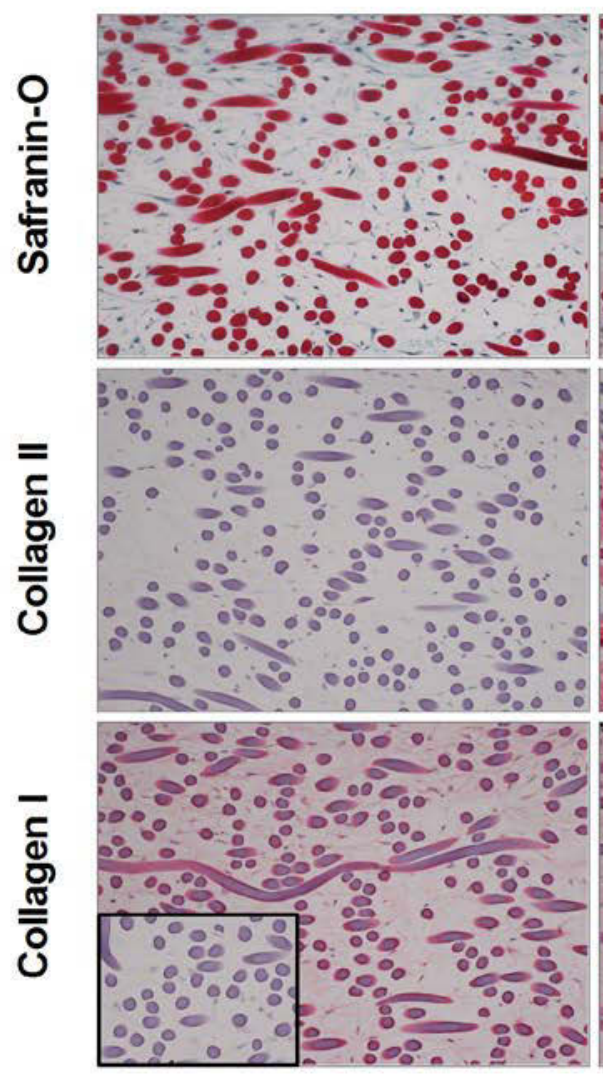

2D + Perfusion
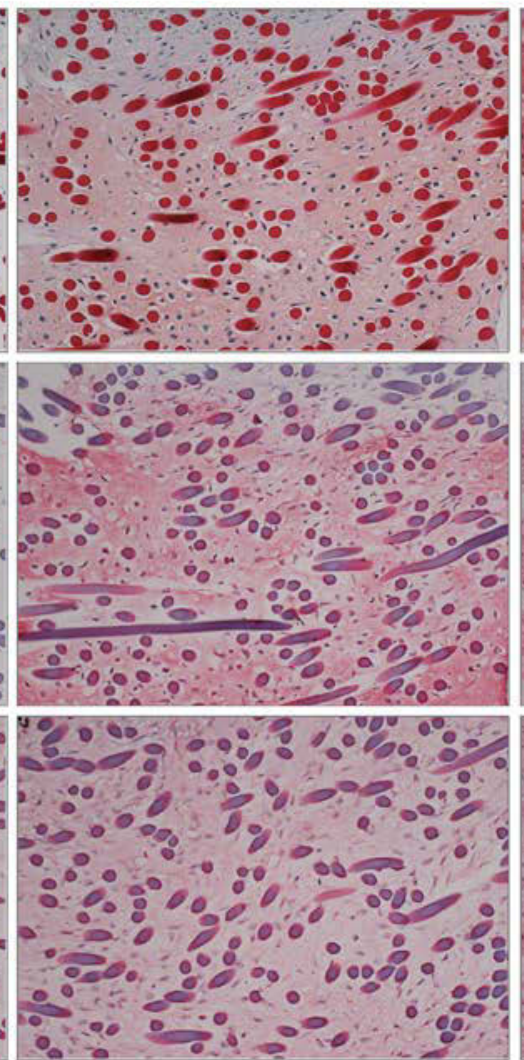

3D + Perfusion

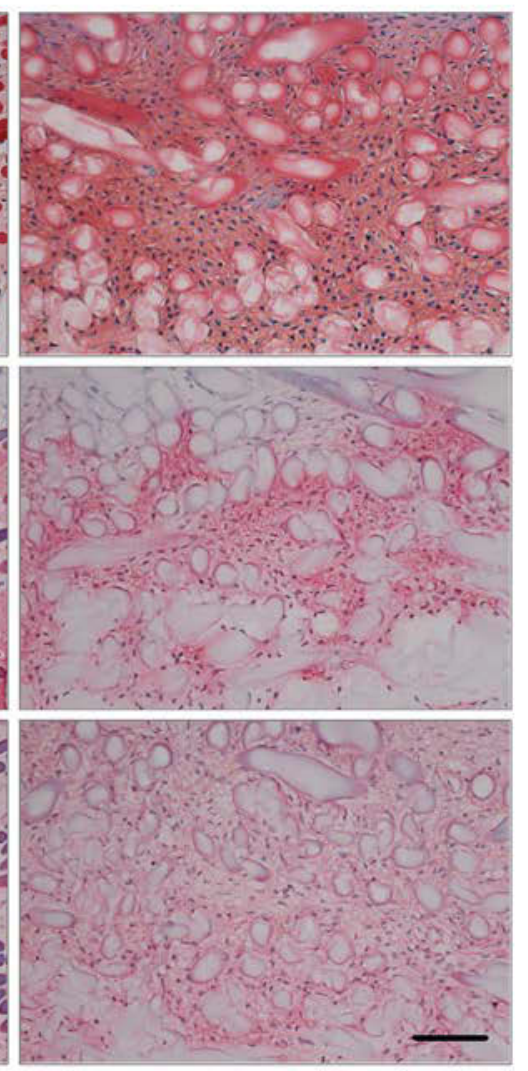

b

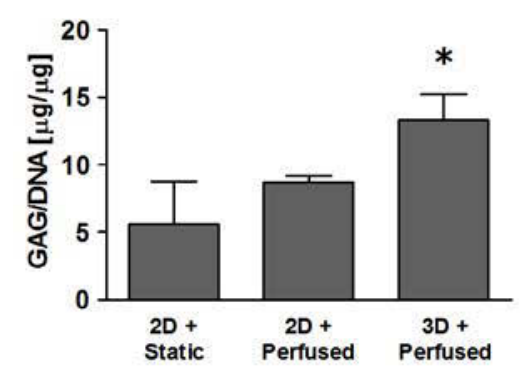

C

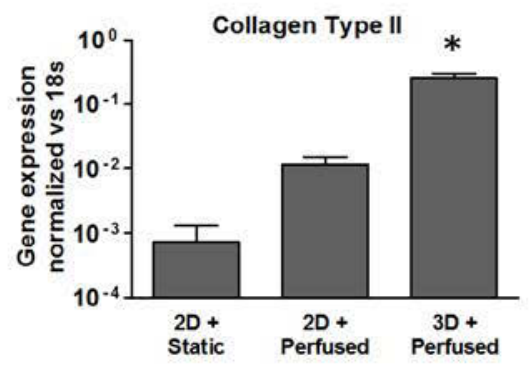

d

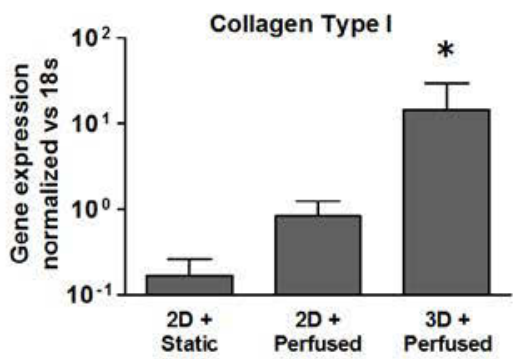

Fig. 4. Engineered constructs following 2 weeks of the differentiation phase. (a) 3D+Perfusion constructs stained slightly more intensely for GAG but had similar staining patterns and intensities for collagen type II and collagen type I than 2D+Perfused constructs. 3D+Perfusion and 2D+Perfusion constructs were more uniformly and intensely stained for GAG and collagen type II than 2D+Static constructs. Note: Fibres of the Hyaff-11 mesh non-specifically stain for Safranin-O, collagen I, and collagen II in 2D+Static and 2D+Perfused constructs. However, likely due to enhanced degradation of the fibres during prolonged perfusion, Hyaff-11 fibres in 3D+Perfused constructs were not stained. The negative control for immunohistochemistry is included as an insert in the image of $2 \mathrm{D}+\mathrm{Static}$ collagen type I staining. Scale bar $=100 \mu \mathrm{m}$. (b) Quantification of GAG indicated that 3D+Perfusion constructs had significantly higher levels of GAG/DNA than the other two experimental groups. (c,d) Expression levels of collagen type II and collagen type I were significantly higher in 3D+Perfusion constructs than for the other two experimental groups. * indicates statistical significance with $p \leq 0.05$.

constructs contained rounded cells embedded within extracellular matrix intensely stained for GAG and collagen type II (Fig. 4a). Compared to 3D+Perfused, constructs perfusion-seeded with $2 \mathrm{D}$ expanded HAC and perfusion cultured for 2 weeks in the bioreactor (2D+Perfusion) stained slightly less for GAG but had similar staining patterns and intensities for collagen type II and I. Constructs generated by the conventional approach (2D+Static) contained cells and matrix stained faintly for GAG and collagen type II at the periphery of the scaffold but contained a large necrotic inner region void of cells and extracellular matrix. All constructs stained positive for collagen type I with similar intensities. Quantification of GAG indicated that 3D+Perfusion constructs contained 
a
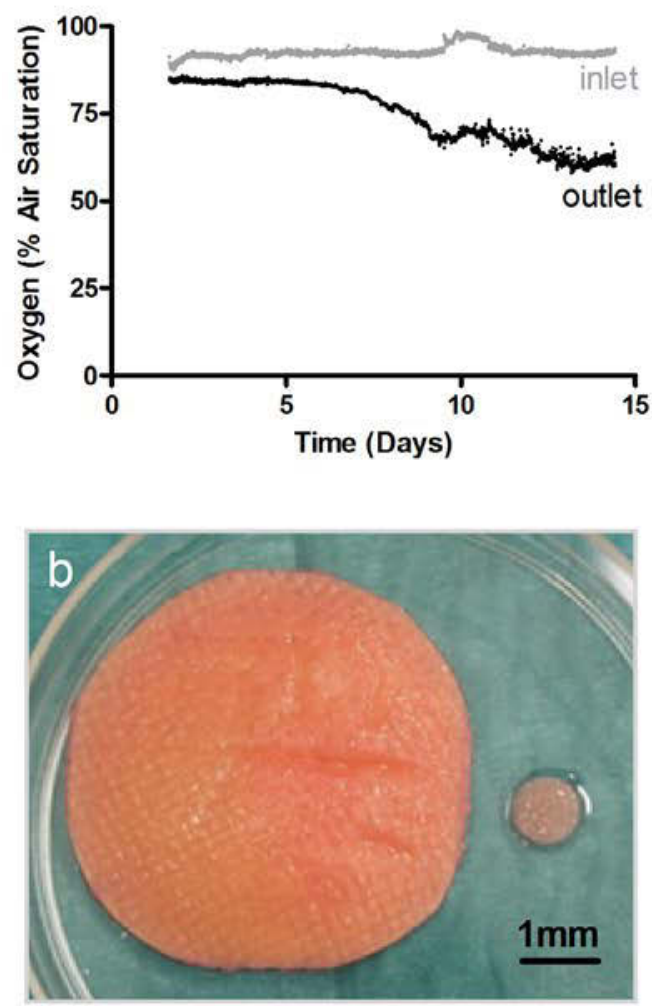

Up-scaled
$2 \mathrm{D}+$ Static

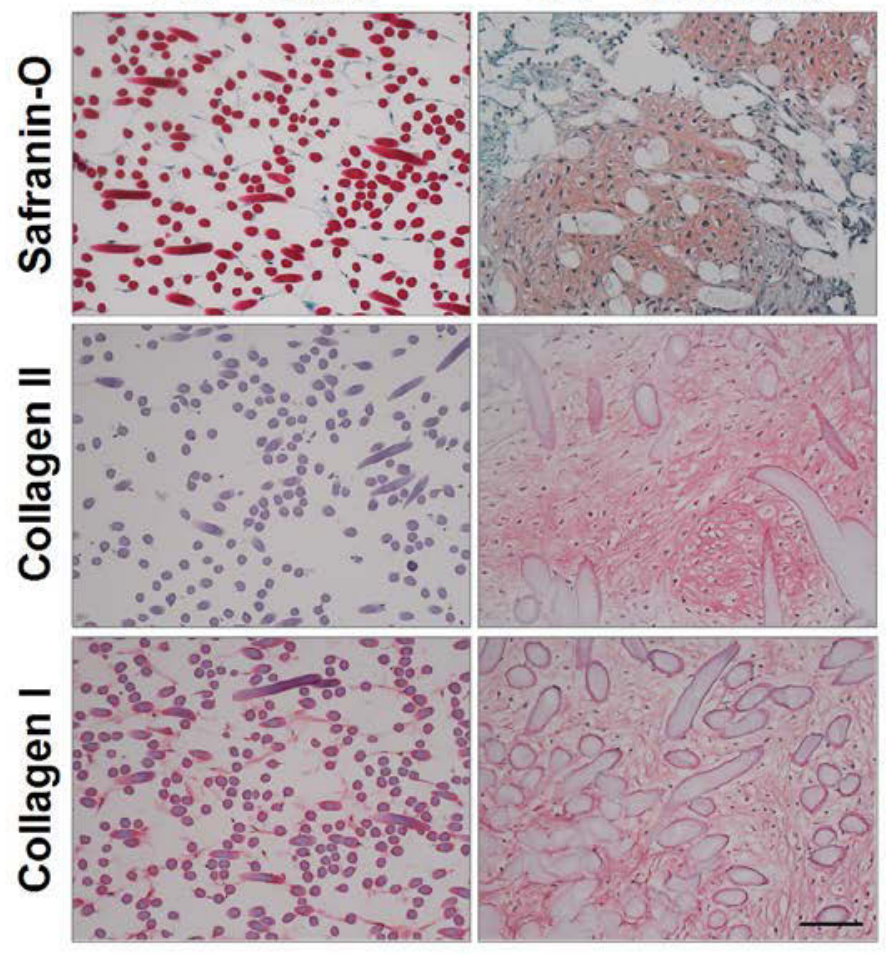

d

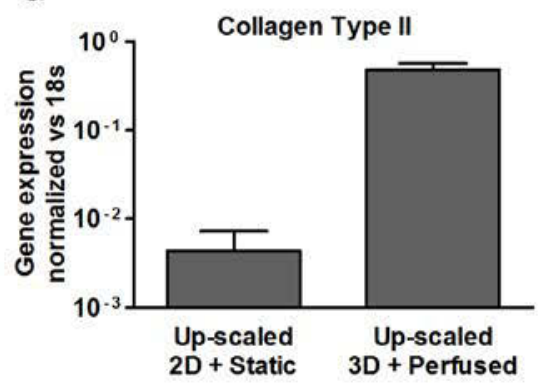

e

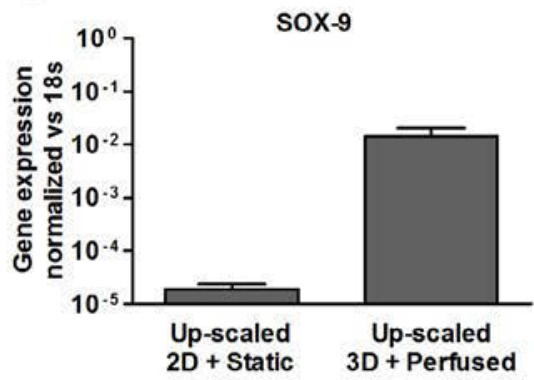

f

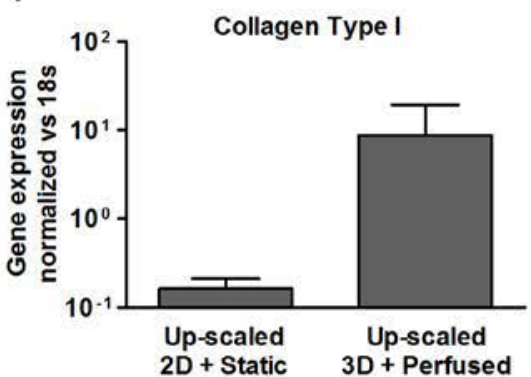

Fig. 5. Up-scaled engineered cartilage grafts. (a) Oxygen profile during the $3 \mathrm{D}$ expansion phase. Oxygen levels in the perfused culture medium were measured at the inlet and outlet of the bioreactor to estimate the number of cells in the 3D construct. (b) Macroscopic appearance of large-scale and small-scale cartilage tissues engineered by the streamlined process. (c) Following the 2 week differentiation phase in the bioreactor, constructs engineered by the streamlined process (Up-scaled 3D+Perfusion) stained positively and rather uniformly for GAG and collagen type II. In contrast, large-scale constructs generated by the conventional manual production procedures (Up-scaled 2D+Static) were highly inhomogeneous and contained large necrotic/void regions. Note: Fibres of the Hyaff-11 mesh non-specifically stain for Safranin-O, collagen I, and collagen II in Up-scaled 2D+Static constructs. However, likely due to enhanced degradation of the fibres during prolonged perfusion, Hyaff-11 fibres in Up-scaled 3D+Perfused constructs were not stained. Scale bar $=100 \mu \mathrm{m}$. (d,e,f) Up-scaled 3D+Perfusion constructs had higher expression of collagen type II, Sox-9, and collagen type I than Up-scaled 2D+Static constructs.

significantly more GAG/DNA $(12.8 \pm 1.7 \mu \mathrm{g} / \mu \mathrm{g})$ than $2 \mathrm{D}+$ Perfusion $(9.0 \pm 0.6 \mu \mathrm{g} / \mu \mathrm{g})$ or $2 \mathrm{D}+$ Static constructs $(6.1 \pm 3.5 \mu \mathrm{g} / \mu \mathrm{g})(p=0.014, p=0.014$; respectively) (Fig. 4b). Expression levels of collagen type II and collagen type I were significantly higher in $3 \mathrm{D}+$ Perfusion constructs as compared to $2 \mathrm{D}+$ Perfusion constructs (CII: 17-fold, $p=0.02$; CI: 86-fold, $p=0.02)$ and 2D+Static constructs (CII: 22-fold, $p=0.02$; CI: 360-fold, $p=0.008$ ) (Fig. 4c,d).

\section{Large-scale cartilage grafts}

Starting from the clinically relevant low cell seeding density, primary HAC could also be extensively expanded directly within the large $50 \mathrm{~mm}$ diameter Hyaff- 11 meshes. The predetermined target cell number of $5 \times 10^{7}$ HAC $\left(8 \times 10^{6}\right.$ cells $\left./ \mathrm{cm}^{3}\right)$ could be reached in 14 to $24 \mathrm{~d}$ of $3 \mathrm{D}$ expansion, depending on the HAC donor. The number of HAC quantified in the constructs at the end of the expansion phase was similar to the number of HAC predicted from 
non-invasive oxygen measurements (Fig. 5a), differing by an average of $11 \% \pm 3 \%$. Considering that we did not observe significant differences in population doublings at $14 \mathrm{~d}$ between 2D Plastic and 3D Hyaff-11 meshes in the small research-scale experiments, 2D population doublings were not assessed during the up-scaled experiments. Following the 2-week phase of differentiation in the upscaled bioreactor, Up-scaled 3D+Perfusion constructs (Fig. 5b) stained positive and rather uniformly for GAG, collagen type II, and collagen type I (Fig. 5c). In contrast, Up-scaled 2D+Static constructs generated by the conventional manual production procedures (i.e. static seeding, 2D expansion, and static culture) were highly inhomogeneous and contained significant necrotic/void regions. In order to quantify the homogeneity/variability of GAG accumulated within the large diameter up-scaled constructs, six specimens were punched out of each construct at various radii and assessed biochemically. The coefficient of variation was calculated among the six specimens punched from each construct and was used as a measure of construct variability. The average GAG/ DNA within Up-scaled 3D+Perfused constructs was 8.34 $\pm 4.27 \mu \mathrm{g} / \mu \mathrm{g}$, with a coefficient of variation of $28 \%$ $\pm 8.5 \%$ among specimens sampled within each construct. The GAG/DNA within Up-scaled 2D+Static constructs was $11.71 \pm 4.27 \mu \mathrm{g} / \mu \mathrm{g}$, with a coefficient of variation of $50 \%$ $\pm 7 \%$ among specimens sampled within each construct. However, as specimens were punched through the crosssection of the constructs, the variability along the depth of the statically cultured constructs (i.e. necrotic centre) is not accounted for by the calculated coefficient of variation. Expression levels of collagen type II and collagen type I were consistent with results obtained in the small-scale model system. Up-scaled 3D+Perfusion constructs had higher expression of collagen type II (3200-fold), Sox-9 (750-fold), and collagen type I (4500-fold) than Up-scaled 2D+Static constructs (Fig. 5 d,e,f).

\section{Discussion}

In this work, we engineered human cartilaginous tissues within a single bioreactor system, from the introduction of primary chondrocytes freshly isolated from a biopsy, until the generation of a cartilaginous tissue graft. We have shown that the limited number of primary chondrocytes that can be isolated from a small cartilage biopsy, in a size that can be obtained during ACI procedures, can be seeded and extensively expanded directly within a 3D scaffold in a perfusion bioreactor system, eliminating the typical labour-intensive manual process of $2 \mathrm{D}$ expansion on plastic flasks/dishes. After switching the operating conditions in the bioreactor to support chondrogenic differentiation, the 3D expanded HAC generated a cartilaginous tissue. Furthermore, we demonstrated that this streamlined tissue engineering process was not only effective to engineer small "research-scale" constructs, but could also be used to generate up-scaled cartilage grafts in a size with clinical relevance.

Previous studies have demonstrated that seeding a high vs. low density of chondrocytes into a scaffold significantly improves chondrogenic differentiation and cartilaginous extracellular matrix deposition (Concaro et al., 2008; Francioli et al., 2010; Moretti et al., 2005). Therefore, in most cartilage tissue engineering approaches, chondrocytes are extensively expanded in monolayer culture using manual methods in order to obtain sufficient quantities of cells prior to seeding into 3D scaffolds. As an alternative to expanding cells in flasks, a number of studies have assessed the expansion of chondrocytes in $3 \mathrm{D}$ bioreactors using collagen-based microcarriers as substrates for proliferation. While extensive proliferation on microcarriers has been achieved by seeding chondrocytes that were previously expanded in 2D on plastic (Bouchet et al., 2000; Frondoza et al., 1996; Surrao et al., 2011), rather limited expansion (four doublings in four weeks) was reported when primary chondrocytes were seeded and cultured on microcarriers (Schrobback et al., 2011; Schrobback et al., 2012). Early studies had also indicated that 2D expanded chondrocytes showed some limited signs of redifferentiation when cultured on microcarriers (Bouchet et al., 2000; Frondoza et al., 1996). However, a number of recent studies have reported that microcarrier-expanded chondrocytes have a rather limited chondrogenic capacity (e.g. ECM production, chondrogenic markers) (Pettersson et al., 2011), which was similar (Schrobback et al., 2011; Surrao et al., 2011) or even lower (Schrobback et al., 2012) than that of 2D expanded chondrocytes.

In our work, we have demonstrated that HAC could be extensively expanded under perfusion in a 3D mesh while maintaining a high chondrogenic capacity to generate a cartilaginous extracellular matrix. Primary HAC were expanded directly within the 3D scaffold until reaching a sufficiently high cell density before inducing subsequent chondrogenic differentiation. The target cell number for expansion was determined from the cell density previously reported for cell seeding of Hyaff-11 meshes for the manufacturing of cartilage grafts for clinical use (based on seeding 2D expanded cells) (Albrecht et al., 2011). In subsequent experiments, we have also observed that by seeding 10-fold fewer primary HAC and extending the duration of the proliferation phase, HAC could be expanded on the 3D Hyaff-11 meshes to at least 9 population doublings (data not shown). This opens the perspective of applying the streamlined process for applications when only very small cartilage biopsies can be obtained. We have shown that HAC expanded directly in 3D Hyaff-11 meshes better maintained a chondrogenic phenotype than those expanded for the same culture time on 2D plastic. To better understand the influence of the Hyaff-11 material itself on HAC proliferation, chondrocytes were also expanded on 2D membranes of Hyaff-11. HAC expanded on 2D Hyaff-11 also maintained a more chondrogenic phenotype than when expanded on 2D plastic, however, the effects were less pronounced as compared to expansion on 3D Hyaff-11 meshes under perfusion. While these results may indicate that the biomimetic hyaluronan-based Hyaff-11 material may better support the maintenance of a chondrogenic phenotype, since HAC proliferated slower on 3D Hyaff-11 meshes than on 2D plastic, we cannot exclude that the higher expression of chondrogenic genes was the result of fewer population doublings. Despite the slower 
proliferation on 3D Hyaff-11 meshes, in all experiments we could obtain a sufficient density of cells to support subsequent chondrogenic differentiation.

Engineered constructs generated by the streamlined process $(3 \mathrm{D}+$ Perfusion) were significantly more cartilaginous and maintained a more chondrogenic phenotype than constructs generated by conventional manual processes $(2 \mathrm{D}+$ Static). The observed differences could be due to the more uniform cell seeding distribution resulting from perfusion vs. static seeding, to a better maintenance of the post-expansion differentiation capacity of HAC when expanded in 3D Hyaff-11 meshes, and/or to better controlled culture conditions under perfusion (e.g. enhanced mass transfer) during the differentiation phase. We have previously shown that the static culture of a $3 \mathrm{D}$ construct, which was previously uniformly seeded by perfusion, results in a necrotic central region (Wendt et al., 2006). This demonstrated that even if a uniform distribution of cells was obtained initially, perfusion was necessary to maintain the uniform cell distribution and to generate homogenous extracellular matrix during prolonged culture. In this study, when HAC were first expanded on 2D plastic prior to seeding, we observed that $2 \mathrm{D}+$ Perfused constructs were significantly more cartilaginous and had significantly higher expression of collagen II mRNA than 2D+Static constructs, suggesting a significant role of the perfusion bioreactor compared to static conditions during the differentiation phase. These results are consistent with a number of previous studies showing enhanced cartilaginous matrix deposition and/ or chondrogenic differentiation following the perfusion culture of 3D constructs based on 2D expanded cells (Alves da Silva et al., 2010; Concaro et al., 2009; Davisson et al., 2002; Forsey et al., 2012; Shahin and Doran, 2011; Tigli et al., 2011; Wendt et al., 2006). However, 3D+Perfused constructs stained only slightly more intensely for GAG than 2D+Perfused constructs and with similar intensity for collagen types I and II. Taken together, this suggests that performing the differentiation culture phase in the bioreactor had a greater impact on the quality of the engineered tissues than the expansion phase performed in either 3D or 2D. Despite using human-derived cells and a short differentiation culture time of only 2 weeks, we have shown that hyaline-like cartilage was generated in 3D+Perfused and 2D+Perfused constructs. While animalderived chondrocytes are still commonly used for tissue engineering studies, it is generally accepted that adult human-derived chondrocytes have a far lower chondrogenic capacity than chondrocytes derived from animals. In addition, while other studies commonly report lengthy differentiation culture times of 4 weeks, 6 weeks, or longer, we aimed to establish a process to generate cartilaginous grafts that minimised the culture time with the ultimate perspective of minimising the manufacturing operating costs in costly good manufacturing practice (GMP) facilities. Although we have focused our investigation on characterising the main extracellular matrix components of cartilage (i.e. GAG and collagen II), future work should also assess if 3D expansion of HAC could influence the RNA expression or protein levels of other extracellular matrix molecules (e.g. aggrecan core protein, small leucine-rich proteoglycans, and other collagen types) or of markers of chondrocyte dedifferentiation (e.g. versican, CD90 (Diaz-Romero et al., 2008)).

After verifying the efficacy of the streamlined cartilage engineering process for generating the small sized 3D+Perfused constructs (i.e. a size typically reported for research purposes), we aimed to further demonstrate the potential applicability of the process to clinical applications by manufacturing large-scale cartilaginous grafts. We previously developed an up-scaled perfusion bioreactor system to engineer up-scaled human cartilage grafts in a size with clinical relevance (Santoro et al., 2010). Using this bioreactor, we reported that tissues grown in the bioreactor were viable and homogeneously cartilaginous. However, constructs engineered in the bioreactor were based on 2D expanded HAC, and therefore required three weeks of manual monolayer cell culture, limiting the potential regulatory and economic benefits of a bioreactorbased manufacturing process. In the current work, we now show the proof-of-principle that implementing the streamlined process with the up-scaled bioreactor we could engineer large-scale constructs (Up-scaled 3D+Perfused) that were more cartilaginous, more uniform, and had significantly higher expression of collagen II mRNA than large-scale constructs generated by conventional manual processes (Up-scaled 2D+Static). Although Up-scaled $2 \mathrm{D}+$ Static had higher ratios of GAG/DNA, it would be misleading to conclude that this method produced more cartilaginous tissues without considering the inhomogeneous GAG deposition and void/necrotic regions within these constructs. This is likely due to the challenge of homogeneously seeding a scaffold with a large surface area, as well as to mass transport limitations to the centre of the construct. Nevertheless, Up-scaled 3D+Perfused constructs stained less intensely for cartilaginous extracellular matrix components than the smaller-scale $3 \mathrm{D}+$ Perfused constructs. Although not quantified in this study, it is possible that the amount of GAG and collagen type II released into the culture medium was higher in the up-scaled bioreactor system. Therefore, as optimisation steps are typically required when scaling-up a bioprocess, future work will be aimed at refining the bioreactor design (e.g. scaffold chamber dimensions) and optimising the streamlined process (e.g. controlled at defined oxygen and $\mathrm{pH}$ levels) for generating large-scale cartilage grafts. With the perspective of clinical translation, the use of GMP compliant materials (e.g. autologous serum instead of bovine serum) must also be assessed when optimising the streamlined process. Based on the procedures established for an ongoing Phase I clinical trial conducted by our laboratory (Pelttari et al., 2014), a sufficient volume of autologous serum could be obtained for the bioreactorbased approach presented here.

While the bioreactor-based process imparts a higher level of control over physicochemical culture parameters as compared to the conventional methods, an operatordependent process may allow for well-established inprocess controls that could be challenging to implement within a bioreactor system. During conventional 2D expansion, a technician can assess cell proliferation by simply bringing a tissue culture flask to the microscope and 
observing the extent of cell growth. Within the bioreactor, cells cannot be observed on the 3D scaffold using such common methods. Therefore, to indirectly monitor cell growth within the bioreactor throughout the proliferation phase, we implemented an oxygen sensor based method to non-invasively estimate the cell number within the $3 \mathrm{D}$ scaffolds. The method, previously developed for smallscale constructs engineered with 2D expanded HAC (Santoro et al., 2011), allowed us to accurately estimate the number of cells within the large-scale constructs, and thus, to determine when the target number of expanded cells had been reached in order to initiate the subsequent differentiation phase. When implemented within a manufacturing process for generating human cartilage grafts for clinical applications, this method could serve as a crucial in-process control and to facilitate compliance with regulatory guidelines.

Based on the promising in vitro results presented in this work, cartilage grafts have been generated by our streamlined process in a semi-automated bioreactor-based manufacturing system and are currently being assessed in a large animal study. As cartilage-cartilage integration is a crucial issue for durable long-term joint repair, it will be important to assess the capacity of the implanted engineered graft to integrate with the surrounding native tissue. Moreover, as the grafts have been implanted into a weight bearing site (medial and lateral condyle), we aim to verify whether the "in vivo bioreactor" will drive the remodelling of the engineered grafts into a tissue with the particular structure and composition of native cartilage. Finally, as the chondrocytes used in this study were obtained from cartilage of cadaveric joints, the efficacy of the streamlined approach should be further validated with cells derived from a biopsy that was harvested from an injured joint.

While manufacturing strategies based on conventional manual cell-culture techniques may ultimately be faced with significant regulatory and commercial challenges, automated and closed bioreactor-based production systems have the potential to overcome the limitations associated with conventional manufacturing methods (Martin et al., 2009). As an alternative to conventional manual methods, bioreactors that automate and streamline the individual steps of a graft manufacturing process can minimise the amount of manual processing labour required, which could have a profound impact on production costs. In-process monitoring techniques and data management systems, which automatically collect and archive process data, could also significantly reduce labour costs associated with quality control and quality assurance. The use of a closed system bioreactor increases the safety of the process and thereby minimises product loss due to contamination. As cross-contamination risks are minimised, closedsystems also allow greater throughput within a single controlled manufacturing area, increasing the efficiency of costly facility infrastructure. Moreover, considering that cell-based tissue engineered products are currently manufactured within very costly Class-B and Class-A clean room facilities, closed-system bioreactors could potentially be operated under less stringent clean room standards in Class-C (Asnaghi et al., 2014). This would have considerable impact on production costs by reducing the building, maintenance, and operating costs of the GMP facility ( 10-fold higher for Class-B compared to Class-C), labour costs (e.g. time for sterile dressing), and capital equipment costs (clothes, equipment, and environmental controls). Taken together, as a result of increased product quality, regulatory compliance, and economic viability of the engineered tissue grafts, the implementation of streamlined bioreactor-based manufacturing processes will likely play a key role in the future translation of these novel therapeutic approaches for wide-spread clinical use.

\section{Conclusions}

We have shown that the small number of primary chondrocytes, which could potentially be obtained from a clinically relevant sized cartilage biopsy, can be extensively expanded directly within a 3D porous scaffold within a perfusion bioreactor system, bypassing the conventional method of 2D cell expansion on plastic dishes. After subsequently switching the bioreactor culture conditions to support chondrogenic differentiation, the 3D expanded HAC generated a cartilaginous tissue. Therefore, human cartilaginous tissues could be engineered within a single bioreactor system, from the introduction of primary chondrocytes freshly isolated from a biopsy, until the generation of a cartilaginous tissue graft. Streamlined and robust tissue engineering processes, as the one we describe here, facilitate the establishment of a simple, compact, and closed bioreactor-based production system, which may be key for regulatory compliant and cost-effective manufacturing of cartilage grafts for clinical applications.

\section{Acknowledgements}

We would like to acknowledge the European Union for financial support ("STEPS"; FP6- \#NMP3CT-2005-500465, www.stepsproject.com). We wish to confirm that there are no known conflicts of interest associated with this publication and there has been no significant financial support for this work that could have influenced its outcome.

\section{References}

Albrecht C, Tichy B, Nurnberger S, Hosiner S, Zak L, Aldrian S, Marlovits S (2011) Gene expression and cell differentiation in matrix-associated chondrocyte transplantation grafts: a comparative study. Osteoarthritis Cartilage 19: 1219-1227.

Alves da Silva ML, Martins A, Costa-Pinto AR, Costa P, Faria S, Gomes M, Reis RL, Neves NM (2010) Cartilage tissue engineering using electrospun PCL nanofiber meshes and MSCs. Biomacromolecules 11: 3228-3236.

Asnaghi MA, Smith T, Martin I, Wendt D (2014) Bioreactors: enabling technologies for research and 
manufacturing. In: van Blitterswijk C, de Boer J (eds) Tissue Engineering, 2nd ed. Chapter 12, Academic Press/ Elsevier, Amsterdam, pp 393-426.

Barbero A, Ploegert S, Heberer M, Martin I (2003) Plasticity of clonal populations of dedifferentiated adult human articular chondrocytes. Arthritis Rheum 48: $1315-$ 1325 .

Bouchet BY, Colon M, Polotsky A, Shikani AH, Hungerford DS, Frondoza CG (2000) Beta-1 integrin expression by human nasal chondrocytes in microcarrier spinner culture. J Biomed Mater Res 52: 716-724.

Brittberg M, Lindahl A, Nilsson A, Ohlsson C, Isaksson O, Peterson L (1994) Treatment of deep cartilage defects in the knee with autologous chondrocyte transplantation. N Engl J Med 331: 889-895.

Concaro S, Nicklasson E, Ellowsson L, Lindahl A, Brittberg M, Gatenholm P (2008) Effect of cell seeding concentration on the quality of tissue engineered constructs loaded with adult human articular chondrocytes. J Tissue Eng Regen Med 2: 14-21.

Concaro S, Gustavson F, Gatenholm P (2009) Bioreactors for tissue engineering of cartilage. Adv Biochem Eng Biotechnol 112: 125-143.

Davisson T, Sah RL, Ratcliffe A (2002) Perfusion increases cell content and matrix synthesis in chondrocyte three-dimensional cultures. Tissue Eng 8: 807-816.

Diaz-Romero J, Nesic D, Grogan SP, Heini P, MainilVarlet P (2008) Immunophenotypic changes of human articular chondrocytes during monolayer culture reflect bona fide dedifferentiation rather than amplification of progenitor cells. J Cell Physiol 214: 75-83.

Farndale RW, Buttle DJ, Barrett AJ (1986) Improved quantitation and discrimination of sulphated glycosaminoglycans by use of dimethylmethylene blue. Biochim Biophys Acta 883: 173-177.

Forsey RW, Tare R, Oreffo RO, Chaudhuri JB (2012) Perfusion bioreactor studies of chondrocyte growth in alginate-chitosan capsules. Biotechnol Appl Biochem 59: 142-152.

Francioli SE, Candrian C, Martin K, Heberer M, Martin I, Barbero A (2010) Effect of three-dimensional expansion and cell seeding density on the cartilage-forming capacity of human articular chondrocytes in type II collagen sponges. J Biomed Mater Res A 95: 924-931.

Frank O, Heim M, Jakob M, Barbero A, Schafer D, Bendik I, Dick W, Heberer M, Martin I (2002) Real-time quantitative RT-PCR analysis of human bone marrow stromal cells during osteogenic differentiation in vitro. J Cell Biochem 85: 737-746.

Frondoza C, Sohrabi A, Hungerford D (1996) Human chondrocytes proliferate and produce matrix components in microcarrier suspension culture. Biomaterials 17: 879888.

Hollander AP, Heathfield TF, Webber C, Iwata Y, Bourne R, Rorabeck C, Poole AR (1994) Increased damage to type II collagen in osteoarthritic articular cartilage detected by a new immunoassay. J Clin Invest 93: 17221732.

Jakob M, Demarteau O, Schafer D, Hintermann B, Dick W, Heberer M, Martin I (2001) Specific growth factors during the expansion and redifferentiation of adult human articular chondrocytes enhance chondrogenesis and cartilaginous tissue formation in vitro. J Cell Biochem $\mathbf{8 1}$ : 368-377.

Jakob M, Demarteau O, Schafer D, Stumm M, Heberer M, Martin I (2003) Enzymatic digestion of adult human articular cartilage yields a small fraction of the total available cells. Connect Tissue Res 44: 173-180.

Martin I, Wendt D, Heberer M (2004) The role of bioreactors in tissue engineering. Trends Biotechnol 22: 80-86.

Martin I, Smith T, Wendt D (2009) Bioreactor-based roadmap for the translation of tissue engineering strategies into clinical products. Trends Biotechnol 27: 495-502.

Mason C, Hoare M (2006) Regenerative medicine bioprocessing: the need to learn from the experience of other fields. Regen Med 1: 615-623.

Moretti M, Wendt D, Dickinson SC, Sims TJ, Hollander AP, Kelly DJ, Prendergast PJ, Heberer M, Martin I (2005) Effects of in vitro preculture on in vivo development of human engineered cartilage in an ectopic model. Tissue Eng 11: 1421-1428.

Nehrer S, Dorotka R, Domayer S, Stelzeneder D, Kotz $R$ (2009) Treatment of full-thickness chondral defects with hyalograft $\mathrm{C}$ in the knee: a prospective clinical case series with 2 to 7 years' follow-up. Am J Sports Med 37 Suppl 1: $81 \mathrm{~S}-87 \mathrm{~S}$.

Pelttari K, Wixmerten A, Martin I (2009) Do we really need cartilage tissue engineering? Swiss Med Wkly 139: 602-609.

Pelttari K, Pippenger B, Mumme M, Feliciano S, Scotti C, Mainil-Varlet P, Procino A, von Rechenberg B, Schwamborn T, Jakob M, Cillo C, Barbero A, Martin I (2014) Adult human neural crest-derived cells for articular cartilage repair. Sci Transl Med 6: 251 ra119.

Pettersson S, Wettero J, Tengvall P, Kratz G (2011) Cell expansion of human articular chondrocytes on macroporous gelatine scaffolds-impact of microcarrier selection on cell proliferation. Biomed Mater 6: 065001.

Ratcliffe A, Niklason LE (2002) Bioreactors and bioprocessing for tissue engineering. Ann NY Acad Sci 961: 210-215.

Ratcliffe E, Thomas RJ, Williams DJ (2011) Current understanding and challenges in bioprocessing of stem cell-based therapies for regenerative medicine. $\mathrm{Br}$ Med Bull 100: 137-155.

Santoro R, Olivares AL, Brans G, Wirz D, Longinotti C, Lacroix D, Martin I, Wendt D (2010) Bioreactor based engineering of large-scale human cartilage grafts for joint resurfacing. Biomaterials 31: 8946-8952.

Santoro R, Krause C, Martin I, Wendt D (2011) Online monitoring of oxygen as a non-destructive method to quantify cells in engineered 3D tissue constructs. J Tissue Eng Regen Med 6: 696-701.

Schrobback K, Klein TJ, Schuetz M, Upton Z, Leavesley DI, Malda J (2011) Adult human articular chondrocytes in a microcarrier-based culture system: expansion and redifferentiation. J Orthop Res 29: 539-546.

Schrobback K, Klein TJ, Crawford R, Upton Z, Malda J, Leavesley DI (2012) Effects of oxygen and culture 
system on in vitro propagation and redifferentiation of osteoarthritic human articular chondrocytes. Cell Tissue Res 347: 649-663.

Shahin K, Doran PM (2011) Strategies for enhancing the accumulation and retention of extracellular matrix in tissue-engineered cartilage cultured in bioreactors. PLoS One 6: e23119.

Surrao DC, Khan AA, McGregor AJ, Amsden BG, Waldman SD (2011) Can microcarrier-expanded chondrocytes synthesize cartilaginous tissue in vitro? Tissue Eng Part A 17: 1959-1967.

Tigli RS, Cannizaro C, Gumusderelioglu M, Kaplan DL (2011) Chondrogenesis in perfusion bioreactors using porous silk scaffolds and hESC-derived MSCs. J Biomed Mater Res A 96: 21-28.

Wendt D, Marsano A, Jakob M, Heberer M, Martin I (2003) Oscillating perfusion of cell suspensions through three-dimensional scaffolds enhances cell seeding efficiency and uniformity. Biotechnol Bioeng 84: 205-214.

Wendt D, Stroebel S, Jakob M, John GT, Martin I (2006) Uniform tissues engineered by seeding and culturing cells in 3D scaffolds under perfusion at defined oxygen tensions. Biorheology 43: 481-488.

\section{Discussion with Reviewers}

Reviewer II: While increasing culture time would increase the ultimate cost of this strategy, enhancing the maturity of the tissue graft at the time of implantation might improve graft response to mechanical stresses in vivo. An improved graft response could, in turn, decrease the period of reduced weight bearing post-operatively and, perhaps, improve long-term stability of repair. To better guide clinical application of this approach, do you think it is worth performing a cost-benefit analysis focused on the duration of tissue maturation time (i.e. under chondrogenic conditions) in vitro, such as within a large animal model? If so, what parameters should be considered in such an analysis?

Authors: These are excellent points that ultimately must be addressed for successful clinical translation. As a first step, we have recently performed a cost analysis of automated $v s$. conventional manual manufacturing process with varying durations of production (1 to 4 weeks) (Asnaghi et al., 2014). According to our model, although cost savings associated with an automated manufacturing process are relatively small with a short production time of 1 week, the savings become highly significant with increasing production times. This analysis highlighted the benefits of automated manufacturing, particularly for the generation of more developed tissue grafts. With regard to a comprehensive cost-benefit analysis, we fear this could hardly be carried out using an animal model, as likely parameters to be considered (e.g. duration and cost of physiotherapy, hospitalisation time, long-term stability of outcome scores, need for re-operation) are predominantly of a clinical nature.
Reviewer II: You discuss crucial in-process controls that improve graft consistency and facilitate compliance with regulatory guidelines. In addition to sensing oxygen levels around the graft, what other in-process sensors could aid in the proposed process? For example, is it currently possible to incorporate an in-line measure of soluble GAGs not incorporated into the graft (i.e. downstream of the graft)? Authors: Similar to the non-invasive oxygen sensors used in this study, we are currently using $\mathrm{pH}$ sensors to non-invasively monitor $\mathrm{pH}$ in the bioreactor throughout the production process. The $\mathrm{pH}$ sensors could be used for in-process controls to not only monitor, but to maintain the $\mathrm{pH}$ at predefined levels, possibly by feedback driven automated medium exchange. This may not only improve and standardise graft quality, but would provide traceability data to facilitate compliance with regulatory guidelines. In parallel, we are also investigating if the quantity of specific extracellular matrix components (e.g. GAG) released into the culture medium can be correlated to the amounts accumulated within the engineered construct. If such correlations can be validated, we aim to establish non-invasive assessments of graft quality (i.e. for release criteria) in the manufacture of cartilage grafts for clinical applications. However, we are unaware of any methodology to perform these biochemical analyses inline, and therefore, for the time being samples of culture medium are currently assessed off-line.

Reviewer II: Did you notice any changes in graft extracellular matrix composition in the axial direction (i.e. direction of fluid flow)? Do you think that changes to media supplement composition and/or conditioning in the direction of flow could be exploited to create anisotropic grafts that vary in composition with tissue depth, as is the case for normal articular cartilage?

Authors: We did not observe specific spatial organisation of cells or matrix indicative of native cartilage in the engineered tissues. Indeed, our objective is to implement a streamlined process to engineer cartilage tissues that are abundant in cartilaginous extracellular matrix, and which could thus be physiologically remodelled once implanted in vivo into a spatially organised and native-like cartilage tissue. Cartilage grafts generated by our streamlined process are currently being assessed in a large animal study. As the grafts have been implanted into a weight bearing site (medial and lateral condyle), we aim to verify whether the "in vivo bioreactor" of the joint will drive the remodelling of the engineered grafts into a tissue with the particular structure and composition of native cartilage. Nevertheless, the reviewer has raised an interesting point. To address this question, bioreactor-based 3D model systems could be used to better understand the influence of specific biochemical and/or mechanical signals on cartilage development. For example, by integrating oxygen sensors into the perfusion bioreactor, as described here, it would be possible to precisely regulate the fluid flow to generate a defined gradient of oxygen across the construct during culture. The oxygen tensions experienced by chondrocytes at the surface and the deep zone of articular cartilage could 
therefore be simulated in vitro in the perfusion bioreactor to assess a potential effect on anisotropic development.

Reviewer II: The authors have effectively shown that the process of tissue production can be streamlined in order to minimise handling, cost and cell manipulation. Are there further steps that the authors feel could be taken in order to reduce the time taken to develop tissue for implantation or that could be made towards producing an "off the shelf" product for cartilage repair based on this system?

Authors: As discussed in response to Question 2 above, we are currently using $\mathrm{pH}$ sensors to non-invasively monitor $\mathrm{pH}$ in the bioreactor throughout the production process. Preliminary evidence suggests that chondrocyte proliferation and differentiation can be influenced by maintaining the $\mathrm{pH}$ at predefined levels, thereby possibly improving graft quality and/or allowing a reduction the production time. The bioreactor-based streamlined process we have described was developed in order to address challenges associated with the manufacturing of autologous cartilaginous tissue grafts. In addition to the production of living engineered cartilaginous tissues, perfusion bioreactors can also be highly efficient to subsequently devitalise/decellularise the engineered tissues, which could potentially be used as off-the-shelf implants for cartilage repair (reviewed in Bender et al. (2013) and Sutherland et al. (2015)). Cell seeding, differentiation, and decellularisation could be performed within a single-unit closed and automated bioreactor system for the streamlined production of an off-the-shelf cartilage implant.

\section{Additional References}

Benders KE, van Weeren PR, Badylak SF, Saris DB, Dhert WJ, Malda J (2013) Extracellular matrix scaffolds for cartilage and bone regeneration. Trends Biotechnol 31: 169-176.

Sutherland AJ, Converse GL, Hopkins RA, Detamore MS (2015) The bioactivity of cartilage extracellular matrix in articular cartilage regeneration. Adv Healthc Mater 4: 29-39.

Editor's Note: Scientific Editor in charge of the paper:. Martin Stoddart 